\title{
Bifenilos policlorados em arroz e feijão do estado do Rio Grande do Sul
}

\author{
Polychlorinated biphenyls in rice and beans from Rio Grande do Sul state, Brazil
}

\author{
Roberta Cocco ${ }^{I}$ Thiago Guilherme Schwanz ${ }^{I}$ Susana Mohr ${ }^{I}$ Juliana Ceolin ${ }^{I I}$ \\ Ana Cláudia Monteiro Braga ${ }^{I I}$ Nilo Zanatta ${ }^{\text {III }}$ Ijoni Hilda Costabeber ${ }^{\text {IV }}$
}

\section{RESUMO}

Os bifenilos policlorados (PCBs) estão entre os poluentes mais tóxicos presentes no meio ambiente, apresentando os alimentos como principal fonte de exposição humana. Com base nisso, este trabalho teve como objetivo determinar os sete PCBs indicadores de contaminação ambiental em 22 amostras de arroz e 18 de feijão do estado do Rio Grande do Sul, Brasil. Além disso, objetivou-se calcular a ingestão diária estimada de residuos, a partir da contaminação existente no arroz e feijão, assim como determinar o teor de gordura das amostras para correlacioná-lo com os niveis de PCBs. A extração e purificação dos compostos foram realizadas pelo método QuEChERS, seguido de identificação e quantificação por CG-EM. Os PCBs 153 e 101 apresentaram as maiores concentrações médias nas amostras de arroz e feijão, respectivamente. Considerando o somatório dos PCBs, este foi de 4,39ng $\mathrm{g}^{-1}$ para o arroz e de 4,17ng $\mathrm{g}^{-1}$ para o feijão. Quanto à ingestão diária estimada, esta foi de $7,82 \mathrm{ng} \mathrm{kg}^{-1}$ e de 3,14ng $\mathrm{kg}^{-1}$ de peso corporal por dia, para o arroz e o feijão, respectivamente. Em relação a percentual de gordura, o arroz e o feijão apresentaram teores de gordura de 0,32\% e de 1,1\%, respectivamente. No entanto, a correlação com os niveis de PCBs não foi significativa. Os resultados demonstram que o estado do $R S$ apresenta fontes importantes de contaminação desses resíduos químicos persistentes.

Palavras-chave: PCBs, arroz, feijão, ingestão diária estimada.

\section{ABSTRACT}

Polychlorinated biphenyls (PCBs) are among the most toxic pollutants in the environment showing foods as the main source of human exposure. Based on this, this study aimed to determine 7 PCBs indicators of environmental contamination in 22 samples of rice and 18 samples of beans in Rio Grande do Sul state, Brazil. Furthermore, this study aimed to calculate the waste estimated daily intake, starting from the existing contamination in rice and beans, as well as wanted to determine the samples fat content to correlate with levels of PCBs. The extraction and purification of the compounds were performed via QuEChERS method followed by identification and quantification by GC-MS. $P C B s 153$ and 101 showed the highest mean concentration in the samples of rice and bean, respectively. Considering the sum of PCBs in the samples, this was 4.39ng $g^{-1}$ for the rice and 4.17ng $\mathrm{g}^{-1}$ for the beans. In estimated daily intake, this was $7.82 \mathrm{ng} \mathrm{kg}^{-1}$ and 3.14ng $\mathrm{kg}^{-1}$ of body weight per day, for the rice and beans, respectively. Regarding the percentage of fat the rice and beans samples had fat contents of $0.32 \%$ and $1.1 \%$, respectively. However, the correlation with the PCBs levels was not significant. The results demonstrate that the State of RS has important sources of these persistent chemical waste contaminations.

Key words: $P C B$, rice, beans, estimated daily intake.

\section{INTRODUÇÃO}

No final da década de 70 , percebeuse o grande risco proporcionado pelos bifenilos policlorados (PCBs), utilizados na composição de fluidos dielétricos em transformadores e capacitores. Tais substâncias apresentam alta toxicidade e estabilidade, bioacumulam-se no meio ambiente

\footnotetext{
IPrograma de Pós-graduação em Ciência e Tecnologia dos Alimentos, Centro de Ciências Rurais (CCR), Universidade Federal de Santa Maria (UFSM), Santa Maria, RS, Brasil.

${ }^{\text {IIC }}$ urso de Farmácia, Centro de Ciências da Saúde, UFSM, Santa Maria, RS, Brasil.

IIIDepartamento de Química, Centro de Ciências Naturais e Exatas (CCNE), UFSM, Santa Maria, RS, Brasil.

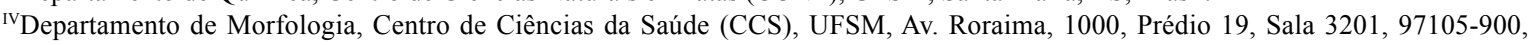
Santa Maria, RS, Brasil. E-mail: ijonicostabeber@gmail.com. Autor para correspondência.
} 
e estão presentes nos alimentos em baixos níveis (VOORSPOELS et al., 2008), porém suficientes para apresentarem efeitos tóxicos ao homem (ZOHAIR et al., 2006). A ingestão através dos alimentos é a principal forma da exposição humana, sendo os alimentos de origem animal os responsáveis por mais de $90 \%$ da ingestão diária de PCBs (BORDAJANDI et al., 2006). Mesmo assim, alimentos de origem vegetal também os contêm (TÖRNKVIST et al., 2011). Referindo-se aos cereais, poucos trabalhos científicos relatam resíduos de PCBs em arroz. No entanto, MARIN et al. (2011) demonstraram contaminação significativa no cereal quando comparada com alimentos de origem animal. Quanto ao feijão, não foram encontrados estudos que reportassem a análise de PCBs neste alimento.

O arroz e o feijão são alimentos básicos para a metade da população mundial. No Brasil, desempenham um importante papel como componente da dieta diária, sendo parte essencial da chamada "cesta básica", distribuída pelo governo à população mais carente (FAO, 2004). Este trabalho teve como objetivo determinar as concentrações de PCBs em amostras de arroz branco polido e feijão preto, coletadas no estado do Rio Grande do Sul, Brasil, no ano de 2010. Adicionalmente, foi determinado o teor de gordura das amostras e calculada a ingestão diária estimada (IDE) de resíduos de PCBs, a partir da contaminação existente.

\section{MATERIAL E MÉTODOS}

Foram analisadas 40 amostras de cereais, sendo 22 de arroz branco polido e 18 de feijão preto, adquiridas em supermercados de cidades do Rio Grande do Sul, Brasil, no ano de 2010. As amostras foram trituradas e armazenadas em freezer a $-4^{\circ} \mathrm{C}$ até a realização das análises. Os padrões dos sete $\mathrm{PCBs}$ indicadores - congêneres 28 (2,4,4'-triclorobifenil), 52 (2,2',5,5'-tetraclorobifenil),

101 (2,2',4,5,5'-pentaclorobifenil),

$118\left(2,3^{\prime}, 4,4^{\prime}, 5\right.$-pentaclorobifenil),

138 (2,2',3,4,4',5'-hexaclorobifenil),

153 (2,2',4,4',5,5'-hexaclorobifenil) e

180 (2,2',3,4,4',5,5'-heptaclorobifenil)

- foram adquiridos de AccuStandard ${ }^{\circledR}$, Inc., New Haven (U.S.A.), com grau de pureza superior a 99,5\%. Como solventes, reagentes e sais, foram utilizados acetonitrila com certificação para análises de resíduos de pesticidas (Mallinckrodt Baker, Inc., NJ, USA), cloreto de sódio (Sigma-Aldrich, Inc., P.O., St. Louis, USA), sulfato de magnésio anidro (Mallinckrodt Baker, Inc., Phillipsburg. Japan), carbono grafitizado 120/400, C-18 (octadecilsilano) e PSA (Primary Secondary Amine) (Supelco Park. Bellefonte, PA, U.S.A).

Para a extração e purificação das amostras, foi utilizado o método QuEChERS, inicialmente proposto por ANASTASSIADES et al. (2003), com algumas modificações. Os extratos foram analisados em um cromatógrafo a gás Agilent Technologies 6890N, acoplado com espectrômetro de massas modelo 5975B inert XL CI/EI MSD. Um microlitro dos extratos foi injetado usando injetor automático split/splitless 7683 Agilent Technologies, no modo splitless. A temperatura deste permaneceu constante a $280^{\circ} \mathrm{C}$. A separação dos PCBs foi realizada em uma coluna capilar de sílica fundida DB-5MS, marca J\&W Scientific, com 30m de comprimento, $0,25 \mathrm{~mm}$ de diâmetro interno e $0,25 \mu \mathrm{m}$ de espessura de fase estacionária. $\mathrm{O}$ hélio foi utilizado como gás de arraste sob pressão inicial de 13,38


A programação da temperatura do forno foi: $60^{\circ} \mathrm{C}$ (1min), $10^{\circ} \mathrm{C} \min ^{-1}$ até $200^{\circ} \mathrm{C}, 5^{\circ} \mathrm{C} \min ^{-1}$ até $225^{\circ} \mathrm{C}$ e $10^{\circ} \mathrm{C} \mathrm{m^{-1 }}$ até $280^{\circ} \mathrm{C}(9,5 \mathrm{~min})$. A temperatura da linha de transferência permaneceu a $290^{\circ} \mathrm{C}$. Para todos os PCBs, foram obtidos espectros e cromatogramas através do modo EI (impacto de elétrons), com energia de $70 \mathrm{eV}$. As temperaturas da fonte e do quadrupolo foram de $230^{\circ} \mathrm{C}$ e $150^{\circ} \mathrm{C}$, respectivamente. $\mathrm{O}$ método operou em modo de monitoramento do íon selecionado (SIM). A identificação e quantificação dos compostos foram realizadas pela comparação dos tempos de retenção dos picos encontrados nas amostras com os tempos de retenção individuais dos padrões de bifenilos policlorados em estudo, sob as mesmas condições de trabalho, bem como pela comparação da abundância dos íons majoritários de cada um dos seis congêneres, obtidos a partir dos padrões de referência, com os íons encontrados na amostra. O método utilizado foi previamente validado, obtendose resultados satisfatórios para todos os parâmetros testados. O coeficiente de variação (CV) para a repetibilidade (precisão intradia) ficou entre 2 e $11 \%$ para as amostras de arroz e entre 14 e $18 \%$ para as de feijão, enquanto que o da precisão intermediária (interdia) ficou entre 16 e 20\% para ambas amostras. Já as recuperações variaram entre 70 e $91 \%$ (arroz) e 71 e $87 \%$ (feijão). O limite de detecção (LD) e o limite de quantificação (LQ) foram os mesmos para as amostras de arroz e feijão, sendo o LD de $0,1 n g \mathrm{~mL}^{-1}$ e o LQ de $0,5 \mathrm{ng} \mathrm{mL} \mathrm{mL}^{-1}$ para todos os PCBs. Brancos foram feitos para cada lote de amostras analisadas, com o objetivo de eliminar possíveis interferentes.

A estimativa da ingestão diária de PCBs através do consumo de arroz e feijão foi calculada 
multiplicando-se a concentração dos resíduos pelo consumo do alimento, dividindo este valor pelo peso corporal médio de $60 \mathrm{~kg}$, sugerido por STORELLI et al. (2011). O consumo médio no Brasil é de 106,85g habitante ${ }^{-1}$ dia $^{-1}$ de arroz e de 45,2g habitante ${ }^{-1}$ dia $^{-1}$ de feijão (CONAB, 2010). Para a determinação da gordura nas amostras de arroz e de feijão, seguiu-se o método descrito por BLIGH \& DYER (1959).

Os dados obtidos foram analisados pelo software Statistica ${ }^{\circledR}$ 7.0. Foram calculadas as médias das concentrações e a frequência de determinação dos congêneres de PCBs no arroz e feijão. Foi realizada a análise de correlação de Spearman para relacionar as concentrações dos PCBs com os níveis de gordura encontrados nos alimentos. As diferenças foram consideradas com no mínimo de $\mathrm{P} \leq 0,05$ de significância.

\section{RESULTADOS E DISCUSSÃO}

$\mathrm{Na}$ tabela 1, apresentam-se os resultados obtidos para as amostras de arroz. O PCB 52 foi o congênere mais detectado, estando presente em $73 \%$ das amostras analisadas. Já os PCBs $153\left(1,43\right.$ ng g $\left.^{-1}\right)$ e $138\left(1,28 \mathrm{ng} \mathrm{g}^{-1}\right)$ apresentaram as maiores médias. O $\sum$ PCBs no arroz foi de 4,39 $\mathrm{ng} \mathrm{g}^{-1}$. BI et al. (2002), ao analisarem amostras de arroz, encontraram maiores contaminações dos PCBs 28, 118 e 138. Com relação ao feijão, os resultados obtidos encontram-se na tabela 2. Observa-se que o PCB 118 foi o congênere mais frequente, estando presente em $22 \%$ das amostras, enquanto que os PCBs 101 $\left(1,63 \mathrm{ng} \mathrm{g}^{-1}\right)$ e $118\left(1,33 \mathrm{ng} \mathrm{g}^{-1}\right)$ obtiveram as maiores médias. O $\Sigma$ PCBs no feijão foi $4,17 \mathrm{ng} \mathrm{g}^{-1}$. Embora os níveis detectados nos dois tipos de alimentos tenham demonstrado diferenças numéricas, estes não apresentaram diferença estatística significativa.
Com relação ao percentual de gordura, o arroz apresentou um nível de $0,32 \%$, enquanto que o do feijão foi superior, com 1,1\%. Esses resultados concordam com os de NEPA/UNICAMP (2006), os quais demonstram que o arroz e o feijão possuem 0,3 e $1,2 \%$ de gordura, respectivamente. Segundo XING et al. (2010), as características da planta, tais como a área de superfície, conteúdo lipídico, composição e arquitetura, podem influenciar nos diferentes níveis de PCBs detectados em vegetais. Correlacionando-se os níveis de PCBs com o percentual de gordura das amostras, a correlação de Spearman não apresentou resultados com diferença estatística significativa, podendo-se supor que a contaminação encontrada está relacionada com o ambiente no qual foram coletadas as amostras.

O arroz e o feijão podem ser usados para identificar resíduos de PCBs em determinadas regiões, porém, por serem plantações temporárias, não absorvem PCBs por um longo período. Um experimento realizado por KACÁLKOWÁ \& TLUSTOS (2011) com alimentos cultivados em áreas cujo solo apresentou níveis consideráveis de PCBs, demonstrou que os alimentos absorvem e bioconcentram estes compostos nas raízes, nos caules e nas folhas, comprovando a presença de contaminação nos alimentos de origem vegetal. Já BI et al. (2002) analisaram a fixação dos PCBs em solos de diferentes regiões de cultivo de arroz e a acumulação desses compostos, observando que as concentrações de PCBs nas amostras da planta não foram diretamente proporcionais às concentrações no solo de cultivo. Porém, os autores detectaram a presença desses compostos tanto no solo como no grão, no caule, na casca e na folha do arroz, com predominância na casca, principalmente, do PCB 28, seguido dos PCBs 101, 108, 118, 138 e 153.

Tabela 1 - Concentrações de bifenilos policlorados $\left(\mathrm{ng} \mathrm{g}^{-1}\right)$ em amostras de arroz do estado do Rio Grande do Sul, Brasil ( $\left.n=22\right)$.

\begin{tabular}{|c|c|c|c|c|c|c|}
\hline PCBs & Média & DP & Mediana & Mínimo & Máximo & $\begin{array}{l}\% \text { de amostras } \\
\text { positivas }(n)\end{array}$ \\
\hline PCB 28 & $<\mathrm{LQ}$ & - & $<\mathrm{LQ}$ & - & - & - \\
\hline РCB 52 & 0,67 & 0,45 & 0,73 & $<\mathrm{LQ}$ & 1,60 & $73 \%(16)$ \\
\hline PCB 101 & 0,24 & 0,52 & 0,05 & $<\mathrm{LQ}$ & 2,19 & $14 \%(3)$ \\
\hline PCB 118 & 0,27 & 0,77 & 0,05 & $<\mathrm{LQ}$ & 3,38 & $9 \%(2)$ \\
\hline PCB 138 & 1,28 & 1,27 & 1,15 & $<\mathrm{LQ}$ & 2,90 & $50 \%(11)$ \\
\hline PCB 153 & 1,43 & 1,31 & 2,27 & $<\mathrm{LQ}$ & 3,11 & $55 \%(12)$ \\
\hline PCB 180 & 0,45 & 1,02 & 0,05 & $<\mathrm{LQ}$ & 3,07 & $14 \%(3)$ \\
\hline$\sum \mathrm{PCBs}$ & 4,39 & & 4,35 & & & \\
\hline
\end{tabular}

n: Número de amostras. DP: Desvio-padrão. LQ: Limite de Quantificação. LD: Limite de Detecção. Valores abaixo do LQ foram considerados como LD/2 para o cálculo da média e mediana. 
Tabela 2 - Concentrações de bifenilos policlorados ( $\left.\mathrm{ng} \mathrm{g}^{-1}\right)$ em amostras de feijão do estado do Rio Grande do Sul, Brasil ( $\left.n=18\right)$.

\begin{tabular}{lllllll}
\hline PCBs & Média & DP & Mediana & Mínimo & Máximo $\%$ de amostras positivas $(n)$ \\
\hline PCB 28 & $<$ LQ & - & $<$ LQ & - & - & - \\
PCB 52 & 0,14 & 0,37 & 0,05 & $<$ LQ & 1,61 & $6 \%(1)$ \\
PCB 101 & 1,63 & 6,71 & 0,05 & $<$ LQ & 28,5 & $6 \%(1)$ \\
PCB 118 & 1,33 & 2,59 & 0,05 & $<$ LQ & 8,58 & $-8,08$ \\
PCB 138 & 0,92 & 2,53 & 0,05 & - & - & - \\
PCB 153 & $<$ LQ & - & $<$ LQ & - & - \\
PCB 180 & $<$ LQ & - & $<$ LQ & & & - \\
$\sum$ PCBs & 4,17 & & 0,55 & & \\
\hline
\end{tabular}

n: Número de amostras. DP: Desvio-padrão. LQ: Limite de Quantificação. LD: Limite de Detecção. Valores abaixo do LQ foram considerados como LD/2 para o cálculo da média e mediana.

$\mathrm{Na}$ literatura consultada, não foram encontrados estudos que reportassem diretamente às concentrações de PCBs em feijão. Também não há limites estabelecidos para PCBs em alimentos de origem vegetal na legislação brasileira, existindo somente limites para produtos de origem animal (BRASIL, 2012). Na China, em um estudo realizado sobre a exposição alimentar, com base em pesquisa de consumo de alimentos e da cesta básica da população, XING et al. (2010) analisaram os 7 PCBs indicadores, dentre outros, detectando a presença de PCBs em $75 \%$ dos legumes, com uma concentração média de $3,78 \mathrm{ng} \mathrm{g}^{-1}$, valor próximo ao encontrado no arroz e feijão do presente experimento.

A partir do cálculo da ingestão diária estimada, os consumidores de arroz e de feijão do estado do Rio Grande do Sul estão tendo uma ingestão de PCBs de 7,82ng kg-1 $\left(0,00782 \mathrm{ng} \mathrm{g}^{-1}\right)$ de peso corporal por dia a partir do arroz e de $3,14 \mathrm{ng}$ $\mathrm{kg}^{-1}\left(0,0034 \mathrm{ng} \mathrm{g}^{-1}\right)$ de peso corporal por dia a partir do feijão. No entanto, cabe salientar que a ingestão de PCBs não é apenas devido ao consumo de arroz e feijão, mas também ao consumo de outros alimentos, como leite e produtos lácteos, carne e derivados (TÖRNKVIST et al., 2011) e, principalmente, de peixes (MIKLAVCIC et al., 2011), sendo que a IDE total de PCBs a partir dos alimentos consumidos ao dia é superior à relatada neste estudo. Diversos trabalhos relatam índices de IDE. No Rio Grande do Sul, foi determinada a ingestão diária estimada de PCBs por estudantes universitários da cidade de Santa Maria a partir do consumo de queijos industrializados e coloniais, encontrando valores de $1,71 \mathrm{pg} \mathrm{kg}^{-1}$ b.w. (106,5pg pessoa ${ }^{-1}$ ) e $0,34 \mathrm{pg} \mathrm{kg}^{-1}$ b.w. $\left(21,2 \mathrm{pg} \mathrm{pessoa}^{-1}\right)$, respectivamente (SANTOS et al., 2006). Em outros estudos realizados no Rio Grande do Sul, foi relatada a presença de PCBs em alimentos de origem animal (leite, queijos, produtos cárneos e carne). Entretanto, o somatório das concentrações médias dos PCBs 28, 52, 138, 153 e 180 foi de $24,85 \mathrm{ng} \mathrm{g}^{-1}$ de gordura para queijos (SANTOS et al., 2006), de $15,5 \mathrm{ng} \mathrm{g}^{-1}$ de gordura para leites pasteurizados (HECK et al., 2007), de 2,82ng g ${ }^{-1}$ de gordura para carnes e de $31,19 \mathrm{ng} \mathrm{g}^{-1}$ de gordura para salsichas hot dog (COSTABEBER et al., 2006). Segundo a legislação brasileira, estabelecida pelo Ministério da Agricultura, Pecuária e Abastecimento, esses valores estão abaixo dos Limites Máximos de Resíduos (LMR), não apresentando, portanto, riscos à saúde com o seu consumo. Em estudo recente, realizado por MOHR et al. (2011), que utilizaram sangue de cordão umbilical, foi encontrado um valor médio de 5,64ng $\mathrm{mL}^{-1}$ para o $\sum$ PCBs 28, 52, 138, 153 e 180 , demonstrando que a contaminação existente nos alimentos é transmitida para os seres humanos através do consumo de alimentos contaminados. Este dado concorda com os relatados no estudo realizado na Itália, por DONATO et al. (2006), que associaram a presença de altos níveis de PCBs em soro sanguíneo de moradores residentes perto de uma indústria química, com o consumo de alimentos produzidos em áreas contaminadas. Estes dados demonstram a importância do monitoramento da contaminação alimentar por resíduos químicos, especialmente por PCBs. Porém, devido ao reduzido número de trabalhos que analisaram alimentos de origem vegetal, torna-se necessário o desenvolvimento de mais pesquisas na área de resíduos tóxicos neste tipo de alimento, tais como os cereais e as leguminosas.

\section{CONCLUSÃo}

Os resultados encontrados no presente estudo demonstram que o arroz e o feijão consumidos no estado do Rio Grande do Sul apresentam contaminação importante por PCBs. Essa 
contaminação, porém, não diferiu significativamente entre as duas matrizes analisadas. Apesar de o feijão ter apresentado porcentagem maior de lipídios em relação ao arroz, o critério usual de que alimentos com maior teor de lipídios possuem maiores concentrações de PCBs não é válido para todos os alimentos, pois os alimentos analisados não diferiram significativamente entre si.

Quanto a IDE, esta foi superior a partir da contaminção de PCBs presente no arroz, quando comparada com a do feijão. Porém, não existem na legislação brasileira valores estabelecidos para a ingestão diária admissível (IDA) de PCBs a partir do consumo de arroz e feijão pela população brasileira.

Sendo este o primeiro estudo a analisar e demonstrar diretamente os níveis de PCBs em arroz e feijão no Brasil, mais estudos são necessários para que se possa determinar as causas da contaminação em alimentos de origem vegetal. Os dados do presente estudo também poderão servir de subsídio para a elaboração de uma legislação brasileira que estabeleça limites máximos de resíduos de PCBs em alimentos de origem vegetal, assim como definir a ingestão diária admissível de PCBs pela ingestão de cereais e leguminosas, a exemplo do que já existe para alimentos de origem animal.

\section{AGRADECIMENTOS}

R. Cocco, J. Ceolin e I. Costabeber agradecem ao Conselho Nacional de Desenvolvimento Científico e Tecnológico (CNPq) pelas bolsas de Mestrado, Iniciação Científica e de Produtividade em Pesquisa, respectivamente. S. Mohr agradece a Coordenação de Aperfeiçoamento de Pessoal de Nível Superior (CAPES) pela bolsa de Doutorado e A. C. Monteiro Braga agradece ao Programa Institucional de Bolsas de Iniciação Científica (PIBIC), Universidade Federal de Santa Maria (UFSM), CNPq, pela bolsa de Iniciação Científica.

\section{REFERÊNCIAS}

ANASTASSIADES, M. et al. Fast and easy Multiresidue method employing acetonitrile extraction/partitioning and "dispersive solid-phase extraction" for the determination of pesticide residues in produce. Journal of AOAC International, v.86, p.412-431, 2003. Disponível em: <http://www.ncbi.nlm.nih.gov/ pubmed/12723926>. Acesso em: 30 abr. 2013.

BI, X. et al. Movement and retention of polychlorinated biphenyls in a paddy Field of WenTai area in China. Agriculture Ecosystems \& Environment, v.89, p.241-252, 2002. Disponível em: <http:// www.sciencedirect.com/science/article/pii/S0167880901002109\#>. Acesso em: 30 abr. 2013. doi: S0167-8809(01)00210-9.

BLIGH, E.G.; DYER, W.J. A rapid method of total lipid extraction and purification. Canadian Journal of Biochemistry and Physiology, v.37, p.911-917, 1959.
BORDAJANDI, L.R. et al. Organochlorine compounds (PCBs, PCDDs and PCDFs) in Seafish and seafood from the Spanish Atlantic Southwest Coast. Chemosphere, v.64, p.1450-1457, 2006. Disponivel em: <http://www.sciencedirect.com/science/ article/pii/ S0045653506000452\#>. Acesso em: 30 abr. 2013. doi: 10.1016/j.chemosphere.2005.12.059.

BRASIL. Ministério da Agricultura, Pecuária e Abastecimento. Instrução Normativa n.11, de 22 de maio de 2012. Disponível em: <http://www.agricultura.gov.br/arq_editor/IN\%2011-\%202012. pdf>. Acesso em: 30 abr. 2013.

CONAB (COMPANHIA NACIONAL DE ABASTECIMENTO). Acompanhamento as safra brasileira. Quarto Levantamento da Safra 2009-2010. Brasilia, 2010. 39p.

COSTABEBER, I. et al. Levels of polychlorinated biphenyls (PCBs) in meat and meat products from the state of Rio Grande do Sul, Brazil. Food and Chemical Toxicology, v.44, p.1-7, 2006. Disponível em: <http://www.sciencedirect.com/science/article/pii/ S0278691505000207>. Acesso em: 30 abr. 2013. doi: 10.1016/j. fct.2005.01.005.

DONATO, F. et al. Exposure to polychlorinated biphenyls in residents near a chemical factory in Italy: the food chain as main source os contamination. Chemosphere, v.64, p.1562-1572, 2006. Disponível em: <http://www.sciencedirect.com/science/article/pii/ S0045653505013986>. Acesso em: 30 nov. 2013. doi: 10.1016/j. chemosphere.2005.11.057.

FAO (FOOD AND AGRICULTURE ORGANIZATION OF THE UNITED NATIONS). Rice is life, 2004. Italy. Disponível em: <http:// www.sciencedirect.com/science/article/pii/S0308814603005399>. Acesso em: 30 abr. 2013. doi: 10.1016/j.foodchem.2003.10.011.

HECK, M.C. et al. Estimation of children exposure to organochlorine compounds through milk in Rio Grande do Sul, Brazil. Food Chemistry, v.102, p.288-294, 2007. Disponível em: <http:// www.sciencedirect.com/science/article/pii/S0308814606004109>. Acesso em: 30 abr. 2013. doi: 10.1016/j.foodchem.2006.05.019.

KACALKOVA, L.; TLUSTOS, P. The uptake of persistent organic pollutants by plants. Central European Journal of Biology, v.6, p.223-235, 2011. Disponível em: <http://link.springer.com/ article/10.2478\%2Fs11535-010-0116-z?LI=true\#>. Acesso em: 30 abr. 2013. doi: 10.2478/s11535-010-0116-z.

MARIN, S. et al. Congener profile, occurrence and estimated dietary of dioxins and dioxin-like PCBs in foods marketed in the Region of Valencia (Spain). Chemosphere, v.82, p.1253-1261, 2011. Disponível em: <http://www.sciencedirect.com/science/ article/pii/S0045653510014384>. Acesso em: 30 abr. 2013. doi: 10.1016/j.chemosphere.2010.12.033.

MIKLAVCIC, A. et al. Mercury, selenium, PCBs and fatty acids in fresh and canned fish available on the Slovenian market. Food Chemistry, v.124, p.711-720, 2011. Disponível em: $<$ http://www. sciencedirect.com/science/article/pii/S0308814610007508>. Acesso em: 30 abr. 2013. doi: 10.1016/j.foodchem.2010.06.040.

MOHR, S. et al. Determinação de bifenilos policlorados em soro de cordão umbilical através de extração por hidrólise ácida seguida de cromatografia a gás acoplada a um microdetector de captura de elétrons. Química Nova, v.34, p.444-449, 2011. Disponível em: $<\mathrm{http} / / / \mathrm{www}$. scielo.br/pdf/qn/v34n3/15.pdf $>$. Acesso em: $30 \mathrm{abr}$. 2013. doi: 10.1590/S0100-40422011000300015.

Ciência Rural, v.45, n.8, ago, 2015. 
NEPA/UNICAMP (NÚCLEO DE ESTUDOS E PESQUISAS EM ALIMENTAÇÃO/UNIVERSIDADE ESTADUAL DE CANPINAS). Tabela brasileira de composição de alimentos TACO, Versão II. 2.ed. Campinas, 2006. 114p.

SANTOS, J.S. et al. Assessment of polychlorinated biphenyls (PCBs) in cheese from Rio Grande do Sul, Chemosphere, v.65, p.1544-1550, 2006. Disponível em: <http://www.sciencedirect. com/science/article/pii/S0045653506004255>. Acesso em: 30 abr. 2013. doi: 10.1016/j.chemosphere.2006.04.003.

STORELLI, M.M. et al. Polychlorinated biphenyls (PCBs), dioxins and furans (PCDD/Fs): Occurrence in fishery products and dietary intake. Food Chemistry, v.127, p.1648-1652, 2011 Disponível em: <http:/www.sciencedirect.com/science/article/pii/ S0308814611002895> . Acesso em: 30 abr. 2013. doi: 10.1016/j. foodchem.2011.02.032.

TÖRNKVIST, A. et al. PCDD/F, PCB, PBDE, HBCD and chlorinated pesticides in a Swedish market basket from 2005 Levels and dietary intake estimation. Chemosphere, v.83, p.193199, 2011. Disponível em: <http://www.sciencedirect.com/science/ article/pii/ S0045653510014475>. Acesso em: 30 abr. 2013. doi: 10.1016/j.chemosphere.2010.12.042.

VOORSPOELS, S. et al. Dietary PCB intake in Belgium. Environmental Toxicology and Pharmacology, v.25, p.179-182, 2008. Disponível em: <http://www.sciencedirect.com/science/ article/pii/S1382668907001639>. Acesso em: 30 abr. 2013. doi: 10.1016/j.etap.2007.10.013

XING, G.H. et al. Dietary exposure to PCBs based on food consumption survey and food basket analysis at Taizhou, China-the world's major site for recycling transformers. Chemosphere, v.81, p.1239-1244, 2010. Disponível em: <http://www.sciencedirect. com/science/article/pii/S0045653510010477>. Acesso em: 30 abr. 2013. doi: 10.1016/j.chemosphere.2010.09.032.

ZOHAIR, A. et al. Residues of polycyclic aromatic hydrocarbons (PAHs), polychlorinated biphenyls (PCBs) and organochlorine pesticides in organically-farmed vegetables. Chemosphere, v.63, p.541-553, 2006. Disponível em: <http://www.sciencedirect.com/ science/article/pii/S0045653505010866>. Acesso em: $30 \mathrm{abr}$. 2013. doi: 10.1016/j.chemosphere.2005.09.012. 